\title{
Knowledge and Consumption Practices on Probiotics in Selected Low to Middle-income Class Filipino Mothers in an Urban City
}

\author{
Cecile Leah T. Bayaga, PhD, ${ }^{*}$ Marietoni B. Pico, RND, ${ }^{1}$ Jethro lan G. Belano, RND, ${ }^{1}$ \\ Demetria C. Bongga, $\mathrm{PhD}^{1}$ and Alonzo A. Gabriel, $\mathrm{PhD}^{2}$ \\ ${ }^{1}$ Breast Milk Research Laboratory, Department of Food Science and Nutrition, College of Home Economics, \\ University of the Philippines, Diliman, Quezon City, Philippines \\ ${ }^{2}$ Laboratory of Food Microbiology and Hygiene, Department of Food Science and Nutrition, College of Home Economics, \\ University of the Philippines, Diliman, Quezon City, Philippines
}

\begin{abstract}
Objective. Mothers are exposed to various functional food components, including probiotics, which have a direct effect on the quality of their breast milk. Probiotics enhance the microbial composition of breast milk, making it more beneficial for the infant's gut health. This study aimed to explore the extent of knowledge and dietary practices on probiotics among low- to middle-income healthy Filipino mothers in an urban area.
\end{abstract}

Methods. Data was gathered using three focus group discussions (FGD) participated in by low- to middle-income healthy Filipino mothers residing in Barangays West Rembo and Rizal in Makati City, Philippines. Prior to the FGD, preliminary interviews of selected participants and market surveys were done to determine the food items to be included in the FGD questionnaire.

Results. A total of 19 mothers participated in the FGDs. The most common probiotic sources identified were yogurt, fermented milk product (Yakult), miso and tausi. The most consumed probiotic source was fermented milk product $(94.7 \%$ of participants), at least once a month; followed by powdered milk (78.9\%), consumed daily. The most commonly reported influence for consumption of these food items was advertisements from television (89.5\%). About $53 \%$ of the participants related probiotics to better digestion and increased immunity as heard from advertisements and through counselling by physicians. When asked to elaborate on how probiotics influence digestion and immunity, almost all participants had difficulty explaining the benefits.

Conclusion. Although majority of the participants consumed food sources of probiotics, they had limited knowledge on its other non-food sources and benefits. In addition, the consumed food source was confined to the food item that was most advertised on television. Thus, there is a need for health and nutrition experts to exert a stronger influence in the community by disseminating information regarding probiotics.

Key Words: probiotics, focus group discussion, maternal health, infant health, infant nutrition, maternal nutrition

\section{INTRODUCTION}

${ }^{*}$ CLTBayaga, MBPico, DCBongga, and AAGabriel are recipients of the 2020 Outstanding Scientific Paper by the National Academy of Science and Technology (NAST).

Corresponding author: Cecile Leah T. Bayaga, PhD

Breast Milk Research Laboratory

Department of Food Science and Nutrition

College of Home Economics

University of the Philippines Diliman

Diliman, Quezon City 1104, Philippines

Email: ctbayaga@up.edu.ph
Numerous studies in the past have established that pregnant and lactating women need good nutrition in order to provide for the adequate growth and development of their infants. Other than the established nutrients needed by pregnant and lactating mothers (i.e., iron, folic acid, and calcium), the progress of modern food product development and processing has presented an array of functional foods to consumers. Functional food products are new foods with an added value to increase or enhance the nutrients present in the food item in terms of amount and function. ${ }^{1}$ 
Functional foods include, but are not limited to, prebiotics and probiotics.

Probiotics are live microorganisms that give health benefits to the host when administered in adequate amounts. ${ }^{2,3}$ Probiotics play an important role in human health: such as 1) homeostasis of natural microbes in the gut; ${ }^{4}$ ) helping in the synthesis of vitamins and mineral salts by metabolic activity; ${ }^{5}$ 3) strengthening the body's immune function; ${ }^{3}$ and 4) assisting in digestion and reducing vulnerability to digestive diseases and disorders. ${ }^{6,7}$ Common sources of probiotics are: fermented food items, milk products, and yogurts. ${ }^{3,4,6}$ Prebiotics are substances present in food that induce the growth or activity of microorganisms (e.g., bacteria and fungi) in the gastro-intestinal tract that contribute to the well-being of their host.

Currently, there is increasing awareness and familiarity with the concept of probiotics in the scientific community that has prompted efforts to find sources and evidence of their health benefits to humans. It would also be advantageous for the consuming public to be familiar with this functional food component. An important benefit of probiotics is its significant role in the gut microbiome of pregnant and lactating women, and consequently for newborns. Pregnant women generally have decreased microbial diversity and dominated by Lactobacillus spp. Hence, they have increased risk of urogenital tract infection, specifically, bacterial vaginosis that predisposes them to preeclampsia and preterm birth. ${ }^{4}$ Probiotic consumption may protect against preeclampsia, gestational diabetes and vaginal infection. When Lactobacilli strains colonize the vagina, lowering of the $\mathrm{pH}$ and vaginal flora improves by killing pathogens. In addition, the probiotic strains prevents preterm birth by modulating the immune response that interferes with the inflammatory cascade. ${ }^{6}$ Additionally, probiotics were found to potentially enhance cytokine levels that promote embryo development.

During lactation, the transfer of probiotics (such as Bifidobacteria and Lactobacillus) from the mother's breast milk to the infant's gut, results in development of the infant's intestinal microbiota. ${ }^{8,9}$ Aside from allergy prevention, the intestinal microbiota also aids in immune regulation by induction and maintenance of tolerance to environmental antigens and self-antigens.

Studies on knowledge, perception, and use of probiotics have been conducted among hospitalized patients, college students, and health care providers. Previous studies conducted among pregnant and lactating women regarding prebiotics and probiotics focused mainly on its administration as a diet supplement. ${ }^{6}$ Considering the potential benefits of probiotics on the health of mothers and their infants, information on mothers' knowledge and practices can be used to recommend effective and practical interventions on improving their level of familiarity and consumption of probiotics. This study aimed to explore the knowledge and dietary practices regarding probiotics among healthy low-to-middle-income class Filipino mothers in an urban setting.

\section{METHODS}

\section{Study site selection}

Makati was strategically chosen as the location for the study due to its organized and ideal health care system. It is one of the cities in the National Capital Region of the Philippines where the health centers are adequately staffed, including doctors and registered nutritionist-dietitians. It is a highly urbanized area with majority of the residents engaged in non-agricultural jobs. The presence of roads, bridges and railways facilitates access to the health center services.

\section{Study design}

This descriptive qualitative study is part of a larger semi-longitudinal study that aims to establish the effect of maternal dietary intake, including prebiotics and probiotics, on the microbiological and biochemical characteristics of breast milk from 0 to 4 months of lactation.

\section{Participant selection}

Women in any physiological state (pregnant or not, lactating or not), were randomly selected from patients who consulted at the barangay health centers from October to mid-December 2016. With the assistance of the barangay nutrition scholar (BNS) or community nutrition worker $(\mathrm{CNW})$, potential participants were invited and screened for inclusion in the study based on a set of criteria (Table 1).

Table 1. Inclusion Criteria for the Selection of the Participants Participant type Inclusion criteria

\begin{tabular}{ll} 
Pregnant mother & $\begin{array}{l}\text { a. Filipino pregnant woman at } 6 \text { to } 9 \text { months } \\
\text { gestation }\end{array}$ \\
b. Aged 18 to 45 years old \\
c. Permanently residing in Makati City \\
d. Has a healthy pregnancy, that is without \\
present or past underlying conditions such \\
as diabetes, dyslipidemia, cancer, endocrine \\
disorder, and chronic inflammation \\
e. Belongs to the low- to middle-income class \\
\hline Lactating mother & $\begin{array}{l}\text { a. Filipino lactating women from } 0 \text { to } 11 \\
\text { months }\end{array}$ \\
b. Aged 18 to 45 years old \\
c. Permanently residing in Makati City \\
d. Had a healthy pregnancy and delivery, \\
that is without present or past underlying \\
conditions such as diabetes, dyslipidemia, \\
cancer, endocrine disorder, and chronic \\
inflammation \\
e. Belongs to the low- to middle-income class \\
mon-pregnant
\end{tabular}


Pregnant mothers included in the study were in their third trimester as this FGD served as one of the preliminary activities prior to the bigger study mentioned above. The bigger study recruited lactating mothers and were asked for their involvement from 0 to 4 months. Similarly, lactating mothers who were 0 to 11 months post-partum were included in the FGD, because more than $60 \%$ of lactating mothers predominantly breastfeed their children until 11 months and 10.4 months is the upper limit of the mean duration in months of any breastfeeding practice among the regions in the country, according to the 2015 survey by DOST -FNRI. For the non-lactating, non-pregnant mothers who were FGD participants, they should have practiced breast feeding previously to be included in the discussions. Generally, participants should be in their child-bearing age, 18 to 45 years old. Mothers outside this age range are more likely to have health problems during pregnancy and post-partum. ${ }^{10}$ Participants of the FGDs should also be permanent residents in Makati City to ensure that they are receiving the same health care provided by the Makati LGU. This criterion ensures that the participants are likely to be comparable in terms of socio-economic status. Last, participants should be apparently healthy to create a homogenous group according to lifestyle and diet.

In addition to the aforementioned inclusion criteria, participants recruited belonged to low- to middle- income families. Based on the 2015 Family Income and Expenditure Survey, ${ }^{11} 37 \%$ of the country's population belonged to the lower income class while $26 \%$ fall within the lower middleincome class. Considering these facts, the study deemed it appropriate to represent the majority of individuals belonging to these income classes. The results generated could then be applicable not just for the target participants but also to other urban poor areas. Further, the participants included in the study were classified as low- to middle-income class using an arbitrary index set by the research team. Participants were considered to belong to the low-income class if their combined utilities bill (electricity and water) was below $\mathrm{Php}$ 2,000.00. Participants with combined utilities bill amounting to or more than Php 2,000.00 were classified as the middleclass income group.

Prior to the FGD, eligible participants were informed on the time and location of the FGD by the BNS.

\section{Preliminary interview}

A preliminary interview of 199 mothers in Makati City was conducted using a food habits questionnaire. The preliminary interviews were conducted at the health centers of Barangays Rizal, East Rembo, West Rembo, Comembo and Olympia. Mothers who participated in the interviews were recruited by the barangay nutritionists. The research team administered the interviews after receiving nutrition and health counseling from the barangay nutritionist.

Objectives of this preliminary interview were to (1) describe the usual diet of the population to which the participants belonged; (2) identify the markets that will be surveyed; and (3) identify the food items to be included in the FGD questionnaire. We asked participants about the usual cooking methods, types of meat bought, markets visited, and person who prepares the food at home. We also asked about their usual diet, including breakfast, lunch, dinner and snacks. Lastly, we asked the participants the frequency of consumption of identified probiotic food sources.

\section{Market survey}

To learn about potential food sources of probiotics in the FGD that are actually being consumed and available to the participants, a market survey was conducted. The markets visited were identified by the responses given in a preliminary interview using a food habits questionnaire. The five most common markets identified in different barangays were Xyris Talipapa and Palar Talipapa in Barangay Rizal, Tablanza Talipapa at Barangay East Rembo, Comembo Talipapa at Barangay Comembo, and Sacramento Talipapa at Barangay Olympia. These markets were visited together with the assigned community nutrition workers or barangay nutrition scholars (BNS) of the selected areas. (Figure 1)

\section{Data collection}

The research team used FGDs to elicit detailed and holistic description from the responses of the participants. It also created a synergistic effect among the participants wherein deeper responses may be stimulated by the answers of the other members in the group such as the reasons for consuming probiotics as part of the regular diets of mothers.

Prior to the conduct of each FGD, a signed informed consent was obtained from each participant, explaining the study protocol and each important point in the consent form. Once the consent forms were signed, an interviewer-facilitated two-part questionnaire was accomplished by the participants prior to the conduct of the focus group discussion. The first part of the questionnaire gathered socio-demographic information of the participant which included age, address, contact number, occupation, and educational attainment. The second part asked for the respondents' frequency of their consumption of probiotic foods. The identified sources of probiotics in the list based on the preliminary interviews and market surves included fermented milk product (Yakult ${ }^{\mathrm{TM}}$ ), milk, yogurt, chocolates, miso (fermented tofu), tausi (black beans), and local fermented food products such as burong talangka (fermented shore crabs or Varunalitterata), burong mustasa (fermented mustard leaves of Brassica junacea)and angkak (fermented white rice or Oryza sativa).

After the completion of the two-part questionnaire described above, an FGD was conducted for all the study participants. The FGD probed on the usual diet of the participants as well as on food sources of probiotics included in their usual diets. It also gauged the familiarity of the participants with probiotics and their health benefits. The questions asked in the FGD are listed in Table 2. 


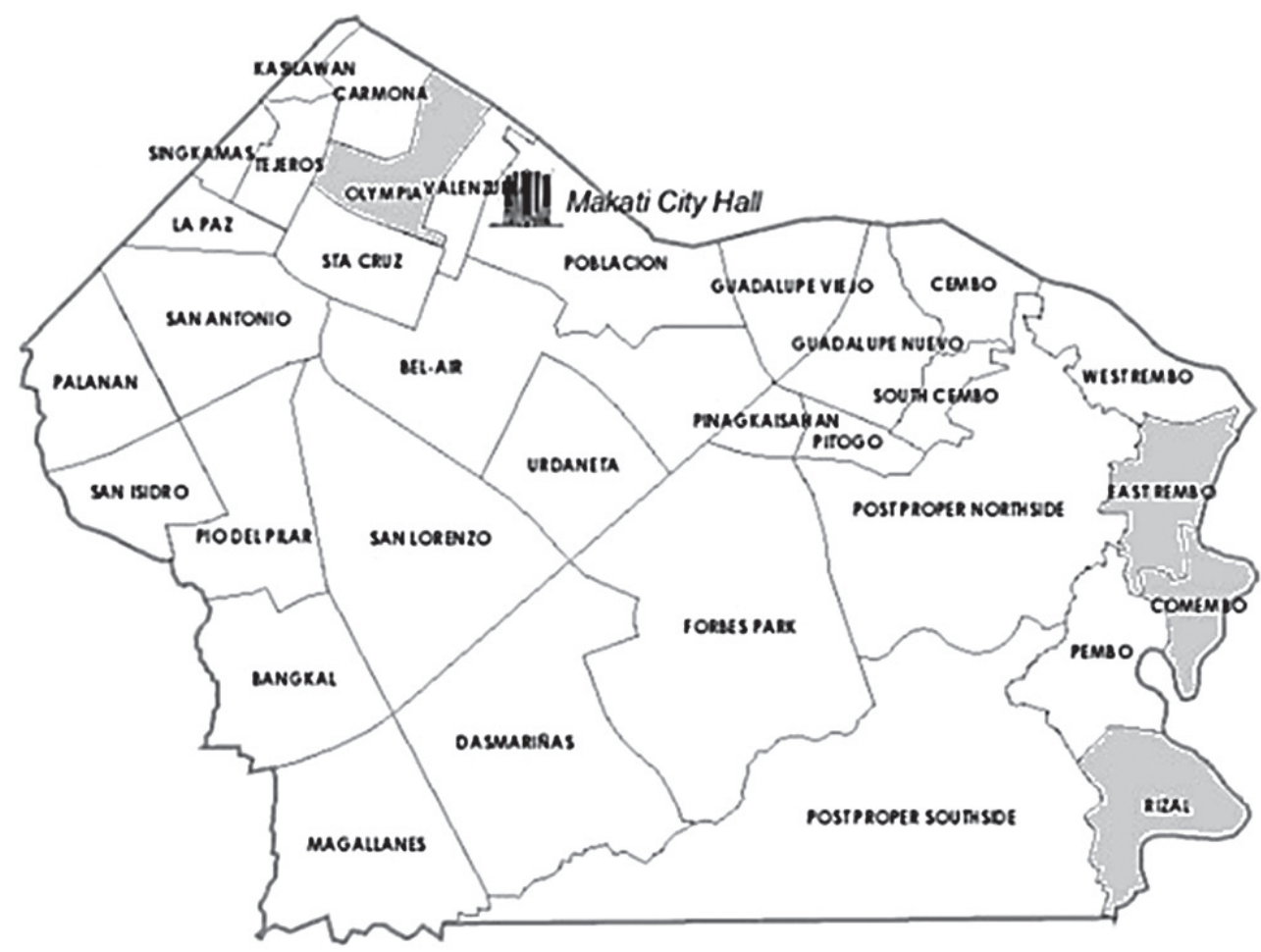

Figure 1. Grayed areas in the map show the barangays in Makati City where the visited markets were located.

Table 2. Questions Asked in the FGD

1. What do you often eat for breakfast? Lunch? Dinner? Snacks?

2. What do you eat/drink the food items listed on the basic information sheet?

3. Who influenced you to eat the food items from the basic information sheet?

4. Have you heard of the word "probiotics"?

5. Why do you consume food items considered as probiotics?

6. When you hear the word probiotics, how do you interpret it?

7. If you were given the chance to eat probiotic items, will you eat them?

After the focus group discussion, the participants were debriefed by giving a brief introduction on what probiotics are and the participants were given the chance to ask questions to the facilitator regarding probiotics.

\section{Data analysis}

The responses from the preliminary interviews were analyzed using descriptive statistics. The food items available from the market surveyed were enumerated and grouped accordingly.

The answers from the FGD were subjected to thematic analysis wherein responses were organized by identifying common concepts, trends, themes, behaviors, terminologies or phrases. During the process of data organization, patterns, connections, relationships and trends that may develop from the responses were noted to substantiate results. Food sources and influences were ranked as per the response of the participant in consideration of their consumption practice.

\section{Ethical clearance}

All volunteers who participated in the focus group discussions gave written informed consent to the protocol, which was approved by the Far Eastern University-Nicanor Reyes Memorial Foundation (FEU-NRMF) Institutional Ethics Review Committee, Diliman, Quezon City (Reference \# FEU-NRMF IERC 2016-0114).

\section{RESULTS}

\section{Development of the FGD questions}

Results from the preliminary interviews revealed that the food items most commonly consumed by all participants that are potential sources of probiotics were powdered milk, chocolate drink and products, miso, tausi, and a popular commercially available probiotic beverage (Yakult). All of these food items were sold in the five markets surveyed (data not shown).

From these results, the probiotic food sources included in the focus group discussion questions were yogurt, milk, fermented foods or buro, chocolate, tausi, miso, and Yakult.

\section{Conduct of focus group discussions}

Nineteen participnts in three FGDs $(6,7$ and 6 participants, respectvely) were included. The distribution and demographic characteristics of participants is shown in Table 3.

The mean (SD) age was similar between the lactating $(28.89 \pm 5.54$ years $)$ and pregnant mothers $(28 \pm 0$ years $)$ 
Table 3. Demographic characteristics of participants of the FGDs $(n=19)$

\begin{tabular}{lccc}
\multicolumn{1}{c}{ Characteristics } & $\begin{array}{c}\text { Lactating mother } \\
(\mathbf{n}=7)\end{array}$ & $\begin{array}{c}\text { Non-pregnant, non-lactating } \\
\text { mother }(\mathbf{n}=6)\end{array}$ & $\begin{array}{c}\text { Pregnant mother } \\
(\mathbf{n}=6)\end{array}$ \\
\hline Age (years, Mean \pm SD) & $28.89 \pm 5.54$ & $47.8 \pm 15.80$ & $28 \pm 0$ \\
Months Pregnant (Mean \pm SD) & - & - & $7.00 \pm 0$ \\
Weeks lactating (Mean $\pm S D)$ & $25.31 \pm 33.66$ & - & - \\
Occupation & & & $4(66.7 \%)$ \\
$\quad$ Stay-at-home mother & $5(71.4 \%)$ & $6(100.0 \%)$ & 0 \\
$\quad$ Self employed & 0 & 0 & $2(33.3 \%)$ \\
$\quad$ Employed outside the home (full time) & $2(28.6 \%)$ & 0 & $6(100.0 \%)$ \\
Civil Status & & & 0 \\
$\quad$ Married & $7(100.0 \%)$ & $6(100.0 \%)$ & 0 \\
$\quad$ Single & 0 & 0 &
\end{tabular}

Table 4. Frequency of consumption of identified food sources of probiotics

\begin{tabular}{lcccc}
$\begin{array}{l}\text { Identified food sources } \\
\text { of probiotics }\end{array}$ & $\begin{array}{c}\text { Lactating } \\
(\mathbf{n}=\mathbf{7})\end{array}$ & $\begin{array}{c}\text { Pregnant } \\
(\mathbf{n}=6)\end{array}$ & $\begin{array}{c}\text { Nonpregnant, nonlactating } \\
(\mathbf{n = 6})\end{array}$ & $\begin{array}{c}\text { Total } \\
(\%)\end{array}$ \\
\hline Yogurt & 4 & 1 & 0 & $5(26.3)$ \\
Yakult & 7 & 6 & 5 & $18(94.7)$ \\
Milk & 4 & 6 & 4 & $14(73.7)$ \\
Fermented foods or buro & 0 & 1 & 0 & $1(5.2)$ \\
Chocolate & 4 & 4 & 4 & $12(63.2)$ \\
Tausi & 0 & 1 & 3 & $4(21.1)$ \\
Miso & 1 & 3 & 3 & $7(36.8)$ \\
\hline
\end{tabular}

but older for the non-pregnant, non-lactating adult women $(47.8 \pm 15.80$ years $)$. Majority of the participants were nonworking $(66.7 \%$ to $100 \%)$. The average gestational age for the pregnant mothers was 7 months while the average lactation period of the lactating mothers was 25 weeks.

From the identified probiotic food sources, the previously specified brand of fermented milk was the most consumed food source $(94.7 \%)$ by majority of the participants and was consumed at least once to twice a month. Powdered milk was the second most consumed food item and was taken on a daily basis by the participants, while the least consumed food item was locally fermented foods or buro (5.2\%). (Table 4)

Majority of the 19 participants who consumed the previously specified brand of fermented milk believed that it helps in good digestion and elimination especially when experiencing constipation during pregnancy. Participants also mentioned that it helps fight bad bacteria, probably gleaned from the advertisement of the branded fermented milk product. However, when the participants were asked to elaborate on how fermented milk product can help in digestion, none of them were able to explain why. In the case of milk, the participants consume it because it is good for the bones and for a healthy body.

The participants who consumed tausi and miso enjoyed their taste and smell; while those who did not, said they were either not familiar or did not like the smell and taste of these fermented foods.

Factors that influenced consumption of probiotics food sources by majority of the respondents (57.9\%), included 1) advise from elders; 2) regular part of home-prepared meals; or 3) seen in television ads (21.1\%).
In terms of familiarity with the term probiotics, a large majority of the respondents $(94.7 \%)$ claimed that they have heard about probiotics from television commercial advertisements and related the term probiotics to the brand of a fermented milk product. Another $10.5 \%$ of the participants attributed the term probiotics to a specific brand of powdered milk.

When asked about their understanding of the term "probiotics", majority of the participants (52.6\%) mentioned that it has something to do with good digestion. In fact, all the participants were one in saying they heard about this from the advertisement of the previously specified brand of fermented milk. Table 5 shows the summary of the responses of the participants on their understanding and interpretation of probiotics.

All the participants in the present study said they were willing to try food sources rich in probiotics if provided by the health care system.

\section{DISCUSSION}

Fermented milk product (Yakult) was the most consumed food source among the identified sources of probiotics. Yakult is a sweetened probiotic milk beverage

Table 5. Responses on the understanding and interpretation of the term "probiotics"

Responses

Frequency (\%)

Good for digestion

$10(52.6)$

Cleanses the digestive system

$1(5.3)$

Strengthens the immune system to prevent sickness

Acts as antibiotic for the body
$2(10.5)$

$1(5.3)$ 
fermented with the bacteria strain Lactobacillus paracasei Shirota, first isolated from the human intestine by Japanese scientist Minoru Shirota in the 1930s. It is sold by Yakult Honsha, based in Tokyo. ${ }^{12}$ On the other hand, the least consumed food item was local fermented foods or buro. Buro is a Filipino term for fermentation where raw ingredients are preserved using a solution of vinegar, salt and other flavorings. In the Philippines, the common food items that are made into buro are mustard leaves (mustasa or Brassica junacea) which is made by fermenting the leaves in brine water for 3 to 5 days; green mangoes (manggang bilaw or Mangifera indica) which is usually fermented with vinegar; and rice (angkak or Oryza sativa) that is usually fermented using vinegar and salt and mixed with different types of fish such as tilapia (Oreochromis niloticus) and mudfish (Channastriata).

Several factors affect the frequency of consumption of the food sources. One of the identified factors was food availability. Most of the participants claimed they can only consume fermented milk product when it is included in their grocery lists. Items like fermented foods were availed of only when supply is brought home from the province where these are produced. Some studies have likewise reported that accessibility influences the consumption of food especially when resources are found in limited places. ${ }^{13}$ Although the present study site was conducted in an urban commercial area, there were limited available food sources of probiotics for the local consumers. This was also evident in the market surveys done prior to the FGDs. The food items sold in all the markets visited were similar, with very little variety, if any. Potential food sources of probiotics items being sold in all these markets included, but were not limited to, fermented milk product, miso, tofu and tokwa (local firm tofu).

Consumption of food items may also depend on consumer acceptability. Food items such as buro may not be as popular as the other food sources because of their peculiar smell and taste, and the respondents were not accustomed to eating these. The sole participant who consumed fermented foods disclosed that she used to eat them in her province, but other members of her family did not like the fermented buro.

Another factor that was observed to influence the consumption of the participants is social environment. An individual is more likely to consume the food items that are also consumed by those around her, according to Vartanian and Herman. ${ }^{13}$ Additionally, individuals tend to consume more of the identified food items when they are told to try these foods by authoritative persons such as physicians..$^{14,15}$ Pregnant and lactating mothers have a better health-seeking behavior since they are naturally conformed to the changes brought about by pregnancy and childbirth. ${ }^{16}$ This behavior makes them more open to follow recommendations from health experts such as medical doctors, dietitians, nurses and midwives.

Another factor identified is the influence of media on the food intake of the participants. Studies have shown that television advertisements have a high impact on the food intake, in that exposure to food advertisements does not only limit its effect on the perception of an individual on the production, but also promotes the consumption of such. ${ }^{15,17}$

There is a shortage of studies exploring the knowledge and consumption practice of probiotics among mothers. Mostly, studies that have been conducted were done among medical students, medical practitioners and health care providers among others. For example, the study of Amarauche ${ }^{18}$ which assessed the knowledge of medical students and practitioners about prebiotics and probiotics has shown that even medical practitioners have limited knowledge on prebiotics and probiotics and their effects. A similar study by Betz et al..$^{19}$ among hospitalized patients also reported little awareness regarding probiotics although they were unknowingly consuming food sources rich in these. Another similar study conducted in the Philippines by Mejia and Barrion ${ }^{20}$ among college students reported a high awareness on probiotics but majority of the participants in their study were not knowledgeable on the beneficial effect of probiotics. A comparable study on the knowledge, perception and use of probiotics by Oliver et al. ${ }^{21}$ among health care providers observed a high familiarity on probiotics but a small percentage of the participants recommended probiotics to their patients.

The generalizability of the findings of this study is limited due to the small sample size and specific study site chosen. The study was able to meet the expected number of participants for the conduct of FGD, as well as the minimum number of FGDs to effectively identify prevalent themes from the data set. However, at least six focus group discussions among mothers in the same socio-economic status as the target population would have ensured a greater number of themes from the gathered data. ${ }^{22}$ The study was conducted in an urban poor community that has a more organized health care system compared to other areas in Metro Manila. More researches are then needed to determine if the themes generated from the current set of participants are similar to those from other urban or rural poor communities.

Based on the results of the study, mothers seem to have limited understanding of the potential benefits of probiotics, not just for themselves, but also for the infants. Thus, it is important to emphasize and disseminate the benefits of probiotics in the diet to mothers and infants. There is also low priority in recommending probiotics to individuals, which limits the potential of probiotics. Members of the health care system are deemed to be in the best position to promote probiotic consumption, having perceived authority when it comes to community health and wellness. ${ }^{15}$

Based on the study findings, participants were only familiar with the previously specified brand of fermented milk as their source of probiotics. There may be a need to stimulate an interest to revisit other locally available or indigenous probiotic food products such as miso, tausi, and buro. 


\section{CONCLUSION}

Majority of participants consume some kind of probiotic food items as part of their diet. However, there is limited understanding of kinds and benefits of prebiotics and probiotics among the selected mothers in the urban area. Television commercial ads play a significant role in awareness and consumption of probiotic food sources. There is a need for health and nutrition experts to emphasize the potential benefits of probiotic consumption to mothers and infants

\section{Recommendations}

Nutrition and health experts can use the results of the study as baseline for further studies to increase generalizabliity and for intervention planning in the local communities. Familiarity on probiotic food sources can be increased by having information campaigns using publicity materials around the community. The topic on the health benefits of probiotics can also be integrated into the regular nutrition education to be conducted for mothers in local health centers.

\section{Acknowledgments}

The authors wish to thank the Nutrition Office of Makati City for their full support and assistance to the research team.

\section{Statement of Authorship}

CLTBayaga, AAGabriel, and DCBongga conceptualized the study. All authors participated in data collection and analysis, and approved the final version submitted.

\section{Author Disclosure}

All authors declared no conflicts of interest.

\section{Funding Source}

This work was funded by the following: (1) UP Emerging Interdisciplinary Research (EIDR-C08-007.1); (2) UP System Enhanced Creative Work and Research Grant (ECWRG 2016-2-082); (3) Philippine Council for Health Research and Development-Department of Science and Technology; and (4) Nutritionist-Dietitians' Association of the Philippines Foundation. The study did not receive any financial assistance from a private or commercial entity.

\section{REFERENCES}

1. Labrecque J, Doyon M, Bellavance F and Kolodinsky J. Acceptance of functional foods: A comparison of French, American, and French Canadian Consumers. Can J Agr Econ. 2006; 54(4), 647-61.

2. Gibson GR, Hutkins R, Sanders ME, et al. The International Scientific Association for Probiotics and Prebiotics (ISAPP) consensus statement on the definition and scope of prebiotics. Nat Reb Gastroenterol Hepatol. 2017; 14(8), 491-502.
3. Klaenhammer TR, Kleerebezem M, Kopp MV and Rescigno M. The impact of probiotics and prebiotics on the immune system. Nat Rev Immunol. 2012; 12(10), 728-34.

4. Garcia-Mantrana I, Bertua B, Martinez-Costa C and Collado MC. Perinatal nutrition: How to take care of the gut microbiota? Clin Nutr Exp. 2016; 6, 3-16.

5. Mushtaq Q, Arshad M and Rehmar S. Probiotic bacteria isolated from fermented milk. World J. Pharm. Med. Res. 2018; 4(5), 300-6.

6. Baldassarre ME, Palladino V, Amoruso A, et al. Rationale of probiotic supplementation during pregnancy and neonatal period. Nutrients. 2018; 10(11), 1693.

7. Marcobal A, Barboza M, Froehlich JW, et al. Consumption of human milk oligosaccharides by gut-related microbes. J. Agric. Food Chem. 2010; 58(9), 5334-40.

8. Tang MLK, Lahtinen SJ and Boyle RJ. Probiotics and prebiotics: clinical effects in allergic diseases. Curr Opin Pediatr. 2010; 22(5), 626-34.

9. Bridgman SL, Kozyrskyj AL, Scott JA, Becker AB and Azad MA. Gut microbiota and allergic disease in children. Ann Allergy Asthma Immunol. 2016; 116(2), 99-105.

10. Klein J, and Committee on Adolescence. Adolescent Pregnancy: Current Trends and Issues. Pediatrics 2005; 116(1), 281-86.

11. Philippine Statistics Authority. 2015 Family Income and Expenditure Survey. [Internet] 2015. [Cited 28 August 2019] Available from: https://psa.gov.ph/sites/default/files/FIES\%202015\%20Final\%20 Report.pdf.

12. Yakult Honsha Co., Ltd. Creating Further Value in Markets of Asia and Oceania. [Internet] March 2017. [Cited 28 August 2019] Available from: https://www.yakult.co.jp/english/ir/management/ pdf/ar2017.pdf

13. Vartanian LR and Herman CP. Are we aware of the external factors that influence our food intake?. Health Psychol. 2008; 27(5), 533-8.

14. Popkin BM, Duffey K and Gordon-Larsen P. Environmental influences on food choice, physical activity and energy balance. Physiol Behav. 2005; 86(5), 603-13.

15. Spanos S, Vartanian LR, Herman CP and Polivy J. Personality, perceived appropriateness, and acknowledgement of social influences on food intake. Pers Individ Diff. 2015; 87, 110-15.

16. Verbiest SB, Tully KP and Stuebe AM. Promoting Maternal and Infant Health in the 4th Trimester. Zero to Three, March 2017.

17. Halford JCG, Gillespie J, Brown V, Pontin EE and Dovey TM. Effect of television advertisements for foods on food consumption in children. Appetite. 2004; 42(2), 221-25.

18. Amarauche CO. Assessing the Awareness and Knowledge of Probiotics by Healthcare Professionals in Nigeria. Journal of Young Pharmacists. 2016; 8(1), 53-55.

19. Betz M, Uzueta A, Rasmussen H, Gregoire M, Vanderwall C and Witowich G. Knowledge use and perceptions of probiotics and prebiotics in hospitalized patients. Nutr Diet. 2015; 72(3), 261-6.

20. Mejia WB and Barrion ASA. Knowledge on Probiotics of Selected College Students in Laguna State Polytechnic University. Journal of The Nutritionist-Dietitians' Association of the Philippines, 2018; 32, 41-52.

21. Oliver L, Rasmussen H, Gregoire MB and Chen Y. Health Care Providers' Knowledge, Perceptions, and Use of Probiotics and Prebiotics. Top Clin Nutr. 2014; 29(2), 139-49.

22. Guest G, Namey E and McKenna K. How Many Focus Groups are Enough? Building an Evidence Base for Nonprobability Sample Size. Field Methods 2016, 1-20. 